from $2.2 \mathrm{~g} / \mathrm{d}$ to $0.2 \mathrm{~g} / \mathrm{d}$ in women. Mean energy intake from ITFA was likewise decimated, falling from $1.1 \% \mathrm{E}$ to $0.1 \% \mathrm{E}$ in both men and women. Eleven hundred and ninety-three fewer CHD deaths (BEST UI 1,149 - 1,688), were potentially attributable to the ITFA reduction, representing some $11 \%$ of the overall 11,100 CHD mortality fall in Denmark between 1991 and 2007. The greatest attributable mortality falls were seen in the most deprived quintiles (reflecting their bigger reductions in ITFA consumption). Adding ITFA data to the original IMPACTsec model improved the overall model fit from $64 \%$ to $73 \%$. The major contributions to the fall in CHD mortality remained consistent across a wide range of sensitivity analyses.

Conclusion Denmark's mandatory elimination of ITFA was very effective. Furthermore, it may well have accounted for approximately $11 \%$ of the substantial reduction in CHD deaths achieved between 1991 and 2007. The most deprived groups benefited the most, thus reducing inequalities. Adopting the Danish ITFA regulatory approach elsewhere could substantially reduce $\mathrm{CHD}$ mortality while also improving health equity.

\section{OP10 PREVALENCE AND SOCIO-DEMOGRAPHIC ASSOCIATIONS OF DIET AND EXERCISE RISK-FACTORS FOR NON-COMMUNICABLE DISEASES IN BO, SIERRA LEONE}

\begin{abstract}
${ }^{1}$ Tahir Bockarie*, ${ }^{2}$ Maria-Lisa Odland, ${ }^{3}$ Haja Wurie, ${ }^{4}$ Rashid Ansumana, ${ }^{5}$ Joseph Lamin, ${ }^{6}$ Miles Witham, ${ }^{1}$ Oyinlola Oyebode, ${ }^{2}$ Justine Davies. ${ }^{1}$ Warwick Medical School, University of Warwick, Coventry, UK; ${ }^{2}$ Institute of Applied Health Research, University of Birmingham, Birmingham, UK; ${ }^{3}$ College of Medicine and Allied Health Sciences, University of Sierra Leone, Freetown, Sierra Leone; ${ }^{4}$ School of Community Health Sciences, Njala University, Bo, Sierra Leone; ${ }^{5}$ Mercy Hospital Research Laboratory, Mercy Hospital, Bo, Sierra Leone; ${ }^{6}$ NIHR Newcastle Biomedical Research Centre, Newcastle University, Newcastle, UK
\end{abstract}

\subsection{6/jech-2021-SSMabstracts. 10}

Background Little is known about modifiable dietary and physical activity risk factors for non-communicable diseases (NCDs) in Sierra Leone. This information is critical to the development of health improvement interventions to reduce the prevalence of these diseases.

Methods Adults aged 40+ were recruited from 10 urban and 30 rural sub-districts in Bo. We examined risk factors including: $<150$ minutes of moderate and vigorous-intensity physical activity (MVPA) weekly, physical inactivity for $>3$ hours daily, $<5$ daily portions of fruit and vegetables, and salt consumption (during cooking, at the table, and in salty snacks). We used logistic regression to investigate the relationship between these outcomes and participants' socio-demographic characteristics. Results 1,966 eligible participants were included in the study. The prevalence of behavioural risk factors was $83.1 \%$ for $<5$ daily portions of fruit and vegetables; $40.8 \%$ and $91.9 \%$ for adding salt at the table or during cooking, respectively and $30.6 \%$ for eating salty snacks; $22.4 \%$ for MVPA $<150$ minutes weekly, and $43.9 \%$ for being physically inactive $>3$ hours daily. Multivariable analysis showed that urban individuals were more likely than rural individuals to consume $<5$ daily portions of fruit and vegetables (Odds Ratio (OR) 1.06, $95 \%$ Confidence Interval (1.00-1.11)), add salt at the table (OR 1.86 (1.80-1.92)), eat salty snacks (OR 2.03 (1.97-2.11)) and do MVPA <150 minutes weekly (OR 1.17 (1.13-1.22)). Male individuals were more likely to add salt at the table (OR 1.25 (1.21-1.29) and consume salty snacks (OR 1.36
(1.32-1.41)) than female individuals but were less likely to report the other behavioural risk-factors examined). Increasing age was associated with higher odds of eating $<5$ daily portions of fruit and vegetables daily; adding salt at the table; eating salty snacks; doing $<150$ minutes of MVPA per week and physical inactivity $>3$ hours. Generally, people in lower wealth quintiles had higher odds of any of the risk factors than those in the highest wealth quintile.

Conclusion Dietary risk factors for NCDs are highly prevalent, particularly among urban residents in Sierra Leone. Our findings show that forthcoming policies in Sierra Leone need to consider modifiable risk factors for NCDs in the context of urbanisation.

This study used data from Cardiovascular Disease (CVD) Risk Factors in Sierra Leone, which was supported by a Small Grant from the Wellcome Trust, grant number 209921/Z/17/ Z.

\section{OP11 IMPLEMENTING A NATIONAL WEIGHT-REDUCTION LIFESTYLE INTERVENTION TO THE NHS HEALTH CHECKS POPULATION: LONG-TERM OUTCOMES MICRO- SIMULATION MODELLING OF THE NHS DIABETES PREVENTION PROGRAMME}

\footnotetext{
${ }^{1,2}$ Claudia Soiland-Reyes* ${ }^{2}$ Martin O'Flaherty, ${ }^{2}$ Chris Kypridemos. ${ }^{1}$ Research and Innovation Department, Salford Royal NHS Foundation Trust, Salford, UK; ${ }^{2}$ Department of Public Health, Policy and Systems, University of Liverpool, Liverpool, UK
}

\subsection{6/jech-2021-SSMabstracts. 11}

Background Type 2 Diabetes Mellitus (T2DM) and obesity are highlighted as the main reasons for the increase in Non-communicable Disease (NCD) incidence in the last decades. The NHS Diabetes Prevention Programme (NHS-DPP) was rolledout in 2016, intending to reduce or delay T2DM in the population, offering lifestyle advice to reduce weight and HbA1c levels in the at-risk population. In this study, we model longterm health, equity and cost-effectiveness outcomes of a weight-reduction lifestyle intervention when added as part of the NHS Health Checks (NHSHCs) programme.

Methods We used the WorkHORSE tool to model NHSHCs scenarios, powered by the IMPACT $\mathrm{ICD}_{\mathrm{NCD}}$ stochastic dynamic microsimulation. We used $\mathrm{R}$ v4.04. We defined the baseline as the current implementation of the NHSHCs programme. Invite and uptake percentages are based on NHS Digital reports; prescription (pharmacological and smoking cessation) and cost data are based on our work evaluating NHSHCs. We defined three policy scenarios for the weight-reduction lifestyle intervention, modelled using empirical data from the NHSDPP first two years:1) reported annual capacity, $2.5 \%$ mean weight loss as percentage weight in intention-to-treat population, cost per completer $£ 435 ; 2$ ) as Scenario 1) but annual capacity is doubled;3) as Scenario 1) but mean weight loss is $4.0 \%$ (observed in completers population). The simulation period was 2020-2039, attrition rate 20\% and standard UK Treasury discount rates were applied.

Results Preliminary results suggest that in Scenario 1) approximately 2,000 (95\% Uncertainty Interval (UI): -370 to 4300 ) T2DM cases could be prevented or postponed. However, when assumed capacity doubled or a higher weight effect was used although in a smaller group, Scenarios 2) and 3) prevented or postponed 3,500 (95\% UI: 1,300 to 6,200$)$ and 2,000 (95\% UI: $-650,4000)$ T2DM cases respectively. Case years prevented or postponed in each scenario are 16,000 